\title{
Advection Diffusion Problems with Pure Advection Approximation in Subregions
}

\author{
M. J. Gander ${ }^{1}$, L. Halpern ${ }^{2}$, C. Japhet ${ }^{2}$, and V. Martin ${ }^{3}$ \\ 1 Université de Genève, 2-4 rue du Lièvre, CP 64, CH-1211 Genève. Switzerland. \\ martin.gander@math. unige.ch \\ 2 LAGA, Université Paris XIII, 99 Avenue J.-B. Clément, 93430 Villetaneuse \\ France. $\{$ halpern, japhet\}@math.univ-paris13.fr \\ 3 LAMFA UMR 6140, Université Picardie Jules Verne, 33 rue Saint-Leu 80039 \\ Amiens Cedex 1 France. veronique.martin@u-picardie.fr
}

Summary. We study in this paper the model problem of the advection diffusion equation on a region which contains a subregion where it is sufficient to approximate the problem by the pure advection equation. We define coupling conditions at the interface between the two regions which lead to a coupled solution which approximates the fully viscous solution more accurately than other conditions from the literature, and we develop a fast algorithm to solve the coupled problem.

\section{Introduction}

There are two main reasons for coupling different models in different regions: the first are problems where the physics is different in different regions, and hence different models need to be used, for example in fluid-structure coupling. The second are problems where one is in principle interested in the full physical model, but the full model is too expensive computationally over the entire region, and hence one would like to use a simpler model in most of the region, and the full one only where it is essential to capture the physical phenomena. We are interested in the latter case here. In our context of advection diffusion, coupling conditions for the stationary case were developed in [GQSL90]; they are obtained by a limiting process where the viscosity goes to zero in one subregion, and it is held fixed in the other. Other coupling conditions were studied in [Dub93] to obtain a coupled solution which is closer to the fully viscous one.

One is also interested in efficient algorithms to solve the coupled problems. These algorithms are naturally based on iteration by subdomains. While an algorithm was proposed in [GQSL90], no algorithm was proposed in [Dub93] for the coupling conditions approximating the fully viscous solution.

We propose here coupling conditions for the case where we are interested in the fully viscous solution of the time dependent advection diffusion equa- 
tion, and we develop an effective iteration per subdomain algorithm for the coupled problem. After introducing our model problem in Section 2 together with the subproblems, we present the two coupling strategies from [GQSL90] and [Dub93] in Section 3, and introduce a new set of coupling conditions. We then compare the approximation properties of the three sets of coupling conditions to the fully viscous solution in Section 4. In Section 5, we present an iteration per subdomain algorithm from [GQSL90], and introduce new algorithmic transmission conditions which imply our new coupling conditions at convergence and lead to an efficient iteration per subdomain algorithm. We show numerical experiments in one and two spatial dimensions in Section 6.

\section{Model Problem}

We consider the non-stationary advection diffusion equation

$$
\begin{aligned}
\mathcal{L}_{a d} u=f, & & \text { in } \Omega \times(0, T), \\
u(\cdot, 0)=u_{0} & & \text { in } \Omega, \\
\mathcal{B} u=g & & \text { on } \partial \Omega,
\end{aligned}
$$

where $\Omega$ is a bounded open subset of $\mathbb{R}^{2}, \mathcal{L}_{a d}:=\partial_{t}+\mathbf{a} \cdot \nabla-\nu \Delta+c$ is the advection diffusion operator, $\nu>0$ is the viscosity, $c>0$ is a constant, $\mathbf{a}$ is the velocity field, and $\mathcal{B}$ is some boundary operator leading to a well posed problem. In the following we call $u$ the viscous solution. We now assume that the viscous effects are not important for the physical phenomena in a subregion $\Omega_{2} \subset \Omega$, and hence would like to use the pure advection operator $\mathcal{L}_{a}:=\partial_{t}+\mathbf{a} \cdot \nabla+c$ in that subregion. With $\Omega_{1}=\Omega \backslash \bar{\Omega}_{2}$, see Figure 1, this
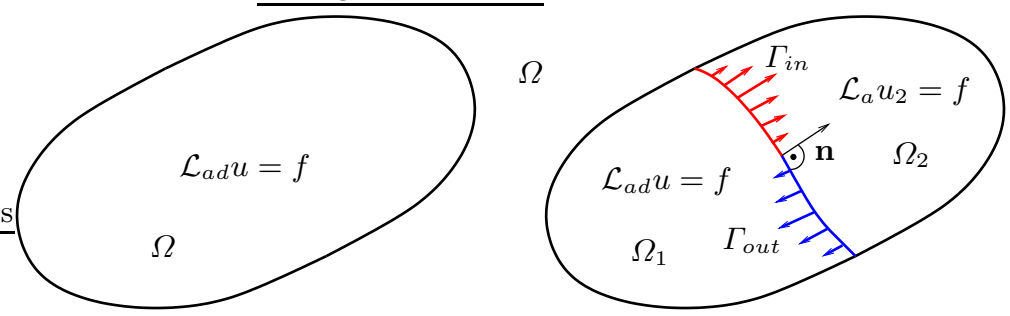

Fig. 1. Fully viscous problem on the left, and coupled subproblems on the right.

leads to the two subproblems

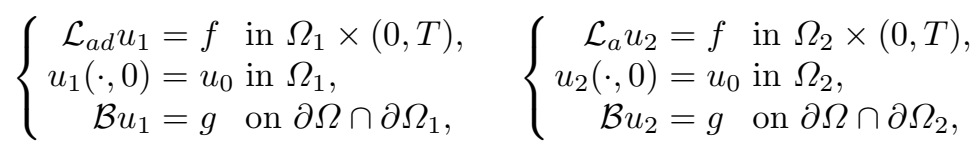

which need to be completed by coupling conditions on $\Gamma$, the common boundary between $\Omega_{1}$ and $\Omega_{2}$. Since the advection operator $\mathcal{L}_{a}$ is of order 1 , it is 
necessary to know on which part of the interface $\mathbf{a} \cdot \mathbf{n}$ is positive or negative (n is the unit outward normal of $\Omega_{1}$ ). We thus introduce $\Gamma_{i n}=\{x \in \Gamma, \mathbf{a} \cdot \mathbf{n}>\mathbf{0}\}$ and $\Gamma_{\text {out }}=\{x \in \Gamma, \mathbf{a} \cdot \mathbf{n} \leq \mathbf{0}\}$, where $\Gamma=\Gamma_{\text {in }} \cup \Gamma_{\text {out }}$, see Figure 1 .

\section{Coupling Conditions}

If we solve the advection diffusion equation in $\Omega$ by a domain decomposition method, it is well-known that the solution as well as its normal derivative must be continuous across $\Gamma$, and the only issue is to define algorithms which converge rapidly to the solution of the global problem, see [QV99] for a review of classical algorithms, and [DO03,Japh98] for optimized ones.

But if the equations are different in each subdomain, there are two issues: first, one has to define coupling conditions so that (2) define together with the coupling conditions a global solution close to the fully viscous one, and second one needs to find an efficient iteration per subdomain algorithm to compute this solution. This algorithm can use arbitrary transmission conditions which are good for its convergence, as long as they imply at convergence the coupling conditions defining the coupled solution.

A first approach to obtain coupling conditions was introduced in [GQSL90] through a limiting process in the viscosity (singular perturbation method). With a variational formulation for the global viscous problem, and letting the viscosity tend to 0 in a subregion, it has been shown in [GQSL90] that the solution of this limiting process satisfies

$$
\begin{aligned}
-\nu \frac{\partial u_{1}}{\partial \mathbf{n}}+\mathbf{a} \cdot \mathbf{n} u_{1} & =\mathbf{a} \cdot \mathbf{n} u_{2} & & \text { on } \Gamma=\Gamma_{\text {in }} \cup \Gamma_{\text {out }}, \\
u_{1} & =u_{2} & & \text { on } \Gamma_{\text {in }},
\end{aligned}
$$

which is equivalent to the coupling conditions

$$
\begin{aligned}
u_{1} & =u_{2} & & \text { on } \Gamma_{\text {in }}, \\
-\nu \frac{\partial u_{1}}{\partial \mathbf{n}} & =0 & & \text { on } \Gamma_{\text {in }}, \\
-\nu \frac{\partial u_{1}}{\partial \mathbf{n}}+\mathbf{a} \cdot \mathbf{n} u_{1} & =\mathbf{a} \cdot \mathbf{n} u_{2} & & \text { on } \Gamma_{\text {out }} .
\end{aligned}
$$

A second set of coupling conditions based on absorbing boundary condition theory was proposed in [Dub93],

$$
\begin{aligned}
u_{1} & =u_{2} & & \text { on } \Gamma_{i n}, \\
\frac{\partial u_{1}}{\partial \mathbf{n}} & =\frac{\partial u_{2}}{\partial \mathbf{n}} & & \text { on } \Gamma_{\text {in }}, \\
-\nu \frac{\partial u_{1}}{\partial \mathbf{n}}+\mathbf{a} \cdot \mathbf{n} u_{1} & =\mathbf{a} \cdot \mathbf{n} u_{2} & & \text { on } \Gamma_{\text {out }} .
\end{aligned}
$$

Both coupling conditions (4) and (5) imply on $\Gamma_{\text {out }}$ that neither the solution nor its derivative are continuous. Since this is in contradiction with the solution of the fully viscous problem, in which we are interested here, we propose a third set of coupling conditions by modifying the conditions (5) to obtain at least continuity of $u$ on the interface, 


$$
\begin{array}{cl}
u_{1} & =u_{2} \quad \text { on } \Gamma_{i n}, \\
\frac{\partial u_{1}}{\partial \mathbf{n}} & =\frac{\partial u_{2}}{\partial \mathbf{n}} \text { on } \Gamma_{i n}, \\
u_{1} & =u_{2} \quad \text { on } \Gamma_{\text {out }} .
\end{array}
$$

In the next section, we show that the coupling conditions (5) and (6) give more accurate approximations to the fully viscous solution than the coupling conditions (4).

\section{Error Estimates with Respect to the Viscous Solution}

We consider the stationary case of (2) on the domain $\Omega=\mathbb{R}^{2}$, with subdomains $\Omega_{1}=(-\infty, 0) \times \mathbb{R}$ and $\Omega_{2}=(0,+\infty) \times \mathbb{R}$, and we estimate the error between the viscous solution and the coupled solution for each of the coupling conditions (4), (5) and (6) when the velocity field a is constant.

Using Fourier analysis and energy estimates, the details of which are beyond the scope of this short paper, we obtain for $\nu$ small the asymptotic results in Table 1 , where $\|\cdot\|_{\Omega_{i}}$ denotes the $L^{2}$ norm in $\Omega_{i}$. These results show that

\begin{tabular}{|l|c|c|}
\hline \multicolumn{3}{|c|}{ Case $\mathbf{a} \cdot \mathbf{n}>0\left(\Gamma \equiv \Gamma_{\text {in }}\right)$} \\
\hline & Conditions (4) & Conditions $(5)$ and $(6)$ \\
\hline$\left\|u-u_{1}\right\|_{\Omega_{1}}$ & $\mathcal{O}\left(\nu^{3 / 2}\right)$ & $\mathcal{O}\left(\nu^{5 / 2}\right)$ \\
\hline$\left\|u-u_{2}\right\|_{\Omega_{2}}$ & $\mathcal{O}(\nu)$ & $\mathcal{O}(\nu)$ \\
\hline
\end{tabular}

\begin{tabular}{|l|c|c|}
\hline \multicolumn{3}{|c|}{ Case $\mathbf{a} \cdot \mathbf{n} \leq 0\left(\Gamma \equiv \Gamma_{\text {out }}\right)$} \\
\hline & Conditions $(4)$ and (5) & Conditions (6) \\
\hline$\left\|u-u_{1}\right\|_{\Omega_{1}}$ & $\mathcal{O}(\nu)$ & $\mathcal{O}(\nu)$ \\
\hline$\left\|u-u_{2}\right\|_{\Omega_{2}}$ & $\mathcal{O}(\nu)$ & $\mathcal{O}(\nu)$ \\
\hline
\end{tabular}

Table 1. Asymptotic approximation quality of the coupled solution to the viscous solution through different coupling conditions.

if $\mathbf{a} \cdot \mathbf{n}>0$, then the approximation of the viscous solution by the coupled solution through conditions (5) and (6) is better on the viscous subregion $\Omega_{1}$ than with the conditions (4). In fact, conditions (5) and (6) are not based on the limiting process in the viscosity, and hence retain in some sense the viscous character of the entire problem. In $\Omega_{2}$ the error is $\mathcal{O}(\nu)$ independently of the coupling conditions, since we solve the advection equation instead of the advection-diffusion equation. Note also that in this case with the coupling conditions (5) and (6) we have continuity of the solution and of its normal derivative, whereas with the coupling conditions (4), we have continuity of the solution only.

If $\mathbf{a} \cdot \mathbf{n} \leq 0$, the solution in $\Omega_{2}$ does not depend on the transmission conditions, and since we solve in this domain the advection equation, the error 
is $\mathcal{O}(\nu)$. Then the error is propagated into $\Omega_{1}$, so we can not have a better error than $\mathcal{O}(\nu)$ in $\Omega_{1}$ independently of the coupling conditions. Note however that now only conditions (6) lead to continuity of the coupled solution.

\section{Algorithmic Transmission Conditions}

We now turn our attention to algorithms to compute the coupled subproblem solution. In [GQSL90], the following algorithm based on the coupling conditions (4) was proposed for the steady case ( $\theta$ is a relaxation parameter to choose)

$$
\begin{gathered}
\left\{\begin{aligned}
& \mathcal{L}_{a d} u_{1}^{k+1}=f \text { in } \Omega_{1}, \\
&-\nu \frac{\partial u_{1}^{k+1}}{\partial \mathbf{n}}=0 \quad \text { on } \Gamma_{\text {in }},
\end{aligned}\right. \\
\left\{\begin{array}{cc}
-\nu \frac{\partial u_{1}^{k+1}}{\partial \mathbf{n}}+\mathbf{a} \cdot \mathbf{n} u_{1}^{k+1}=\mathbf{a} \cdot \mathbf{n} u_{2}^{k} & \text { on } \Gamma_{\text {out }},
\end{array}\right. \\
\left\{\begin{array}{cc}
\mathcal{L}_{a} u_{2}^{k+1}=f & \text { in } \Omega_{2}, \\
u_{2}^{k+1}=\theta u_{1}^{k}+(1-\theta) u_{2}^{k} & \text { on } \Gamma_{\text {in }},
\end{array}\right.
\end{gathered}
$$

and it was shown that the algorithm is well posed and convergent.

In [GHJ02] an algorithm was proposed for the conditions (6) in the steady state case. This algorithm does not use the coupling conditions, but better suited transmission conditions which imply at convergence the coupling conditions. We generalize this approach here to the unsteady case, which leads to an optimized Schwarz waveform relaxation method. We first consider the case of a constant velocity field. If $\mathbf{a} \cdot \mathbf{n} \leq 0$, i.e. $\Gamma \equiv \Gamma_{\text {out }}$, the solution in $\Omega_{2}$ does not depend on the conditions on $\Gamma$, and to obtain (6), Dirichlet conditions must be used for $\Omega_{1}$. Now if $\mathbf{a} \cdot \mathbf{n}>0$, i.e. $\Gamma \equiv \Gamma_{i n}$, then we use the theory of absorbing boundary conditions to obtain optimal transmission conditions $\mathcal{B}_{1}$ and $\mathcal{B}_{2}$ for the algorithm,

$$
\left\{\begin{array} { r l r l } 
{ \mathcal { L } _ { a d } u _ { 1 } ^ { k + 1 } = f } & { } & { \text { in } \Omega _ { 1 } \times ( 0 , T ) , } \\
{ u _ { 1 } ^ { k + 1 } ( \cdot , 0 ) } & { = u _ { 0 } } & { } & { \text { in } \Omega _ { 1 } , } \\
{ \mathcal { B } u _ { 1 } ^ { k + 1 } } & { = g } & { } & { \text { on } \partial \Omega \cap \partial \Omega _ { 1 } , } \\
{ \mathcal { B } _ { 1 } u _ { 1 } ^ { k + 1 } = \mathcal { B } _ { 1 } u _ { 2 } ^ { k } } & { } & { \text { on } \Gamma \times ( 0 , T ) , }
\end{array} \quad \left\{\begin{array}{cll}
\mathcal{L}_{a} u_{2}^{k+1}=f & & \text { in } \Omega_{2} \times(0, T), \\
u_{2}^{k+1}(\cdot, 0)=u_{0} & & \text { in } \Omega_{2}, \\
\mathcal{B} u_{2}^{k+1}=g & & \text { on } \partial \Omega \cap \partial \Omega_{2}, \\
\mathcal{B}_{2} u_{2}^{k+1}=\mathcal{B}_{2} u_{1}^{k} & & \text { on } \Gamma \times(0, T) .
\end{array}\right.\right.
$$

Using the error equations, one can show that if $\mathcal{B}_{1}$ is the advection operator, then we have convergence of the algorithm in two steps.

In the case of a non constant velocity field, we propose to follow precisely the same strategy, which leads to the algorithm

$$
\left\{\begin{array} { r l } 
{ \mathcal { L } _ { a d } u _ { 1 } ^ { k + 1 } } & { = f \text { in } \Omega _ { 1 } \times ( 0 , T ) , } \\
{ u _ { 1 } ^ { k + 1 } ( \cdot , 0 ) } & { = u _ { 0 } \text { in } \Omega _ { 1 } , } \\
{ \mathcal { B } u _ { 1 } ^ { k + 1 } } & { = g \text { on } \partial \Omega \cap \partial \Omega _ { 1 } , } \\
{ \mathcal { L } _ { a } u _ { 1 } ^ { k + 1 } } & { = f \text { on } \Gamma _ { \text { in } } \times ( 0 , T ) , } \\
{ u _ { 1 } ^ { k + 1 } } & { = u _ { 2 } ^ { k } \text { on } \Gamma _ { \text { out } } \times ( 0 , T ) . }
\end{array} \quad \left\{\begin{array}{rl}
\mathcal{L}_{a} u_{2}^{k+1} & =f \text { in } \Omega_{2} \times(0, T), \\
u_{2}^{k+1}(\cdot, 0) & =u_{0} \text { in } \Omega_{2}, \\
\mathcal{B} u_{2}^{k+1} & =g \text { on } \partial \Omega \cap \partial \Omega_{2}, \\
u_{2}^{k+1} & =u_{1}^{k} \text { on } \Gamma_{\text {in }} \times(0, T) .
\end{array}\right.\right.
$$


Note that if the sign of $\mathbf{a} \cdot \mathbf{n}$ is constant, then algorithm (8) converges in two steps like algorithm (7). If not, our numerical results in the next section suggest that the algorithm has good convergence properties also, but it remains to prove convergence of the new algorithm in that case.

\section{Numerical Results}

We first consider the stationary case in $1 \mathrm{~d}$, with parameters $\nu=0.1, c=1$ and $f(x)=\sin (x)+\cos (x)$. In Figure 2, we show on the left the viscous and coupled solutions for $a=1$, and on the right for $a=-1$. The interface $\Gamma$ is at $x=0$, and in each case the boundary conditions are chosen such that there is no boundary layer. One can clearly see that for
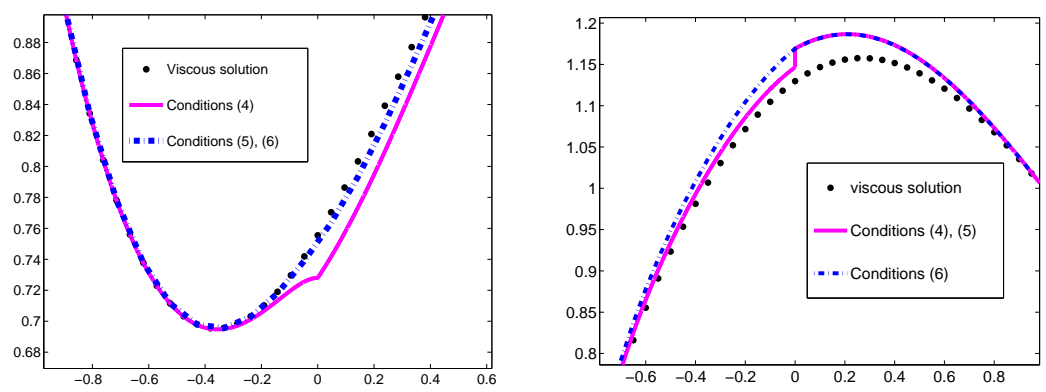

Fig. 2. Viscous and coupled solutions for $a>0$ on the left and for $a<0$ on the right.

$a>0$, conditions (4) lead to a jump in the derivative at the interface, whereas with conditions (6), the coupled solution and its derivative are continuous. For $a<0$, conditions (4) lead to a discontinuity at the interface, whereas conditions (6) lead to a continuous coupled solution. Note that the jump is proportional to $\nu$, see [GQSL90].

In Figure 4, we compare the viscous and the coupled solutions for several values of $\nu$ in the $L^{2}$ norm in $\Omega_{1}$ and $\Omega_{2}$ when $a=1$ and $a=-1$. The numerical results agree well with the theoretical results given in Section 4.

We next consider the time dependent

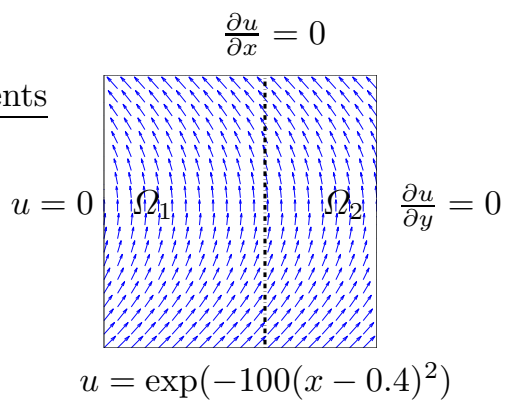

Fig. 3. Domain $\Omega$ case in two dimensions with a rotating velocity, as shown in Figure 3. The viscosity is $\nu=0.001$, we work on the homogeneous equation $f \equiv 0$, and the rotating velocity is given by $a(x, y)=0.5-y$, 

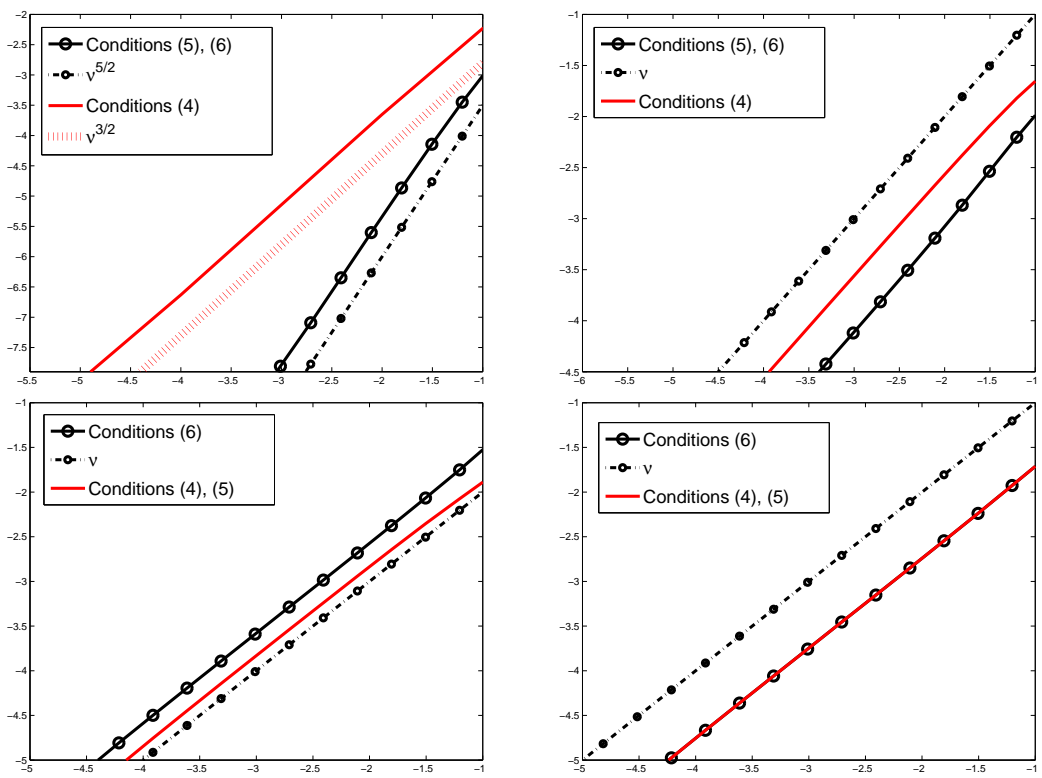

Fig. 4. $1 \mathrm{~d}$ case : $L^{2}$-error for $a=1$ in $\Omega_{1}$ on top left and in $\Omega_{2}$ on top right, and for $a=-1$ in $\Omega_{1}$ at the bottom left and in $\Omega_{2}$ at the bottom right, versus $\nu$.

$b(x, y)=0.5$, such that $\mathbf{a} \cdot \mathbf{n}$ is positive on the first half of the interface and negative on the other half.

Figure 5 shows cross sections of the solution at $y=0.3$ and $y=0.5$ where $\mathbf{a} \cdot \mathbf{n}>0$, and the information goes from $\Omega_{1}$ to $\Omega_{2}$ and stops diffusing after reaching the interface, and then cross sections at $y=0.7$ and $y=0.9$, where $\mathbf{a} \cdot \mathbf{n}<0$, and diffusion sets in again after crossing the interface.

\section{Conclusions}

We have proposed a new set of coupling conditions which permits the replacement of the advection diffusion operator by the pure advection operator in regions where the viscosity is not very important, and which retain better asymptotic approximation properties to the fully viscous solution than earlier coupling conditions in the literature. We have also defined a rapidly converging iteration by subdomain algorithm which uses computational transmission conditions which at convergence imply the new coupling conditions. While numerical experiments show good convergence properties of this new algorithm, it remains to prove convergence of the new algorithm. 

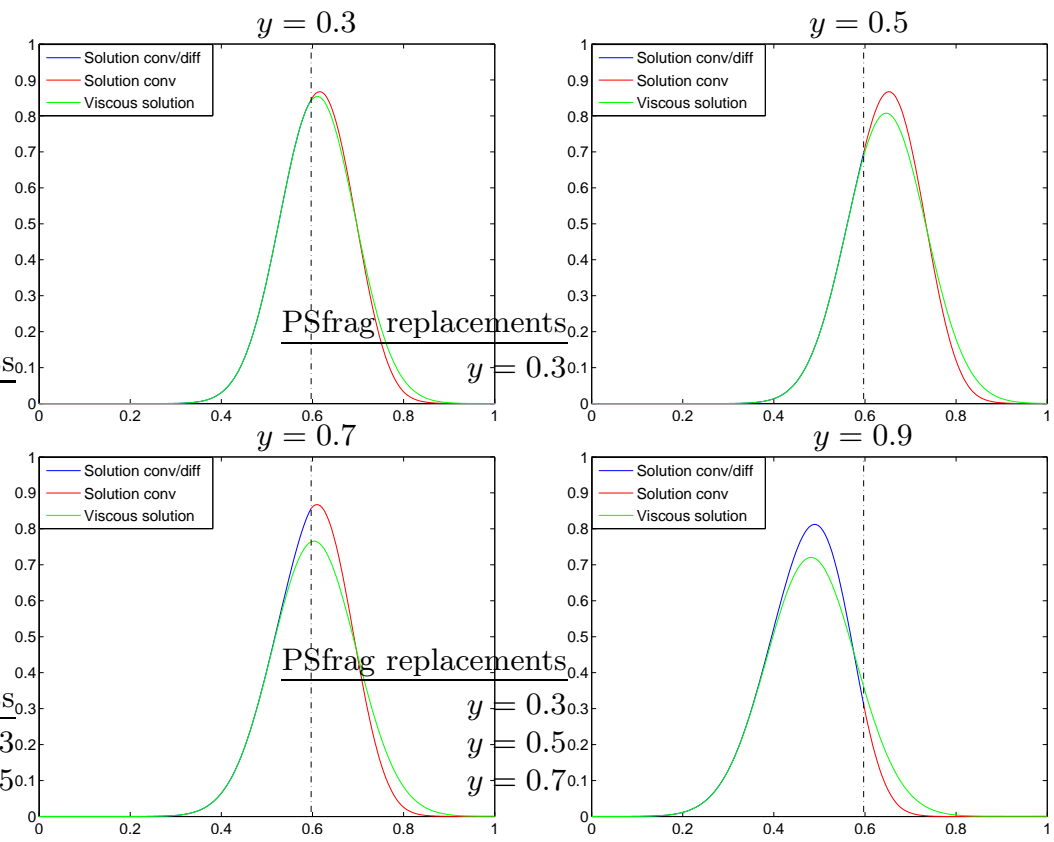

Fig. 5. Cross sections of the viscous and coupled solutions with conditions (6) at the final time for various positions $y$ on the interface $\Gamma$.

\section{References}

[Dub93] Dubach, E., Contribution à la résolution des equations fluides en domaine non borné. PhD thesis. Université Paris 13, (1993).

[DO03] Dubois, O., Optimized Schwarz Methods for the Advection-Diffusion Equation. Master's thesis. McGill University, (2003).

[GHJ02] Gander, M. J., Halpern, L., Japhet, C., Optimized Schwarz algorithms for coupling convection and convection-diffusion problems. Domain decomposition methods in science and engineering (Lyon, 2000), 255-262, Theory Eng. Appl. Comput. Methods, Internat. Center Numer. Methods Eng. (CIMNE), Barcelona, (2002).

[GQSL90] Gastaldi, F., Quarteroni, A., Sacchi Landriani, G., On the coupling of two-dimensional hyperbolic and elliptic equations: analytical and numerical approach. Third International Symposium on Domain Decomposition Methods for Partial Differential Equations (Houston, TX, 1989), 22-63, SIAM, Philadelphia, PA, (1990).

[Japh98] Japhet, C., Méthode de décomposition de domaine et conditions aux limites artificielles en mécanique des fluides: méthode optimisée d'ordre 2. PhD thesis. Université Paris 13, (1998).

[QV99] Quarteroni, A., Valli, A., Domain decomposition methods for partial differential equations, Oxford Science Publications, (1999). 